Thus it appears that while trauma can hardly be identified as a cause of basal, intraventricular, or spinal meningiomas, it may be an important factor in some of the turnours related to the cranial convexities, though in only a small proportion of cases can this be established with reasonable certainty. Sir Francis Walshe, ${ }^{5}$ however, considered that the inferences to be drawn from Cushing and Eisenhardt's admirable, but neglected, monograph were entirely valid and must not be ignored. Walshe described three examples of meningioma in which the tumour could reasonably be related to an earlier injury. In these cases the interval between the injury and the onset of symptoms varied from two months to 32 years, two of the tumours being situated at the pterion and one being parasagittal. Walshe then proceeded to rebuke the "keeping an open mind" attitude of his contemporaries towards this problem with customary verbal felicity: "Surely the perpetually 'open mind' is not an effective instrument of thought, and may too easily become a euphemism for the mind closed to the lessons of experience."

Recently J. Walsh, R. Gye, and T. J. Connelley re-examined the whole subject and added two fresh case reports. The first of these concerned a man who had struck his head on being thrown from a stretcher in the course of an explosion at Tobruk in 1941. He quickly recovered, but a small lump remained in the skin to mark the site of injury. This appeared gradually to increase in size ; in 1967, when he was admitted to hospital, it had reached $6 \mathrm{~cm}$. in diameter. The mass was shown to be bone infiltrated with meningioma. It was successfully removed, together with an underlying large parasagittal meningioma. The second patient, who had been thrown from a motor-cycle in 1940 and struck the back of his head without sustaining any frácture, developed a bony lump at the site of the injury 17 years later. This was removed, and a meningioma en plaque $1 \mathrm{~cm}$. thick was then disclosed, with obliteration of the sagittal sinus.

While it is rare to be able to obtain such close correlation as in these two cases, it is fairly common for patients to offer a past history of trauma to the head in some form or another But, since the criteria for accepting a causal relationship of a meningioma with an old injury must be rigid, it is unlikely often to be established. Caution is clearly required in the interpretation of symptoms, for patients will tend to associate with a knock on the head a hyperostotic intracranial meningioma presenting as a bony lump.

1 Russell, D. S., and Rubinstein, L. J., Pathology of Tumours of the Nervous System, 2nd ed., p. 3. London, E. Arnold, 1963.

2 Bramwell, B., Intracranial Tumours, p. 3, 1888. Edinburgh, Pentland.

3 Cushing, H., and Eisenhardt, L., Meningiomas: Their Classification Regional Behaviour, Life History and Surgical End Results, p. 71. 1938. Springfield, Ill., Thomas.

4 Zülch, K. J., Brain Tumours: Their Biology and Pathology, p. 49. New York, Springer, 1957.

5 Walshe, Sir F., Lancet, 1961, 2, 993.

- Walsh, J., Gye, R., and Connelley, T. J., Medical fournal of Australia, 1969, 1, 906 .

\section{Treatment of Adder Bite}

There are three naturally occurring snakes in Britain. The commonest and largest is the ring snake or grass snake, Natrix natrix, which grows up to $2 \mathrm{~m}$. in length. The smooth snake, Coronella austriaca (up to $75 \mathrm{~cm}$.), is rare and is confined to southern English counties. The third and only venomous variety is the adder, Vipera berus. Adult specimens are $60-80 \mathrm{~cm}$. in length. All three species are usually greyish in appearance, though the colour may vary considerably. As a rule the venomous adder can be easily distinguished by the dark longitudinal zigzag marking along its back. It is widely distributed throughout Britain, occurring in clearings and along the edges of woods, on moors, and on mountains.

When it is considered that $V$. berus is one of the most widely distributed venomous snakes-it is found in a wide belt ranging from Portugal to China-it is remarkable how little has been recorded in medical papers about its venom and the effects of its bite. As with most venomous creatures, the effects depend mainly on the amount of venom injected. Fortunately, $V$. berus bites are no exception to the general rule in snake bite that only a minority of human victims receive enough venom to cause serious poisoning. The majority bitten have slight or no poisoning; they suffer from the effects of fright, and have variable local pain, swelling restricted to below the elbow or knee, and perhaps some nausea. In cases of moderate poisoning vomiting can follow within a few minutes of the bite; it may be associated with abdominal pain and diarrhoea and may be most unpleasant for the victim. Prostration, collapse, and even loss of consciousness may also follow within some minutes. The cause of this rapid collapse (which usually resolves within half an hour) is obscure. Sometimes it may be due to emotional upset, but it has also been experienced by victims such as doctors who would be unlikely to faint through fright. When poisoning is moderate, swelling may spread during the ensuing one or two days to the whole limb, but no further generalized symptoms develop. The swelling with accompanying discoloration resolves without necrosis ${ }^{1}$ within a few weeks. In cases of severe poisoning persistent shock is the main feature. There may be electrocardiographic abnormalities suggesting a myocardial lesion. ${ }^{2}$ In the few studies recorded, coagulation has been normal.

As first-aid treatment the site of the bite should be wiped and covered with a handkerchief or cloth; it should not be incised, as this frequently introduces infection. In Britain the application of a tourniquet serves no useful purpose unless there will be a delay exceeding several hours before hospital can be reached. If available, aspirin or alcohol in moderation is helpful. The victim should be taken to the nearest hospital, resting horizontally so far as is practicable. If a doctor is called to see the patient before hospital is reached, and vomiting is troublesome, an intramuscular injection of chlorpromazine is indicated. The patient should be turned prone to avoid inhalation of vomit. Reassurance of the patient and associates is achieved more by the doctor's calm behaviour than by words.

1 Morton, T. C., British Medical fournal, 1960, 2, 373.

2 Brown, R. and Dewar, H. A., British Heart Fournal, 1965, 27, 144

3 Russell, F. E., Quilligan, J. J., Rao, S. J., and Shannon, F. A., fournal of the American Medical Association, 1966, 195, 596.

British Medical fournal, 1961, 1, 1621.

s Stanic, M., Toxicon, 1969, 6, 287.

- Reid, H. A., British Medical fournal, 1968, 3, 359. 
In hospital only symptomatic treatment is required for slight or moderate poisoning-chlorpromazine for vomiting, analgesics for pain, and an intravenous antihistamine for angioneurotic-type of oedema (rare but recorded). If restlessness is extreme, paraldehyde may be needed. The bitten limb should be supported but otherwise left alone. Antibiotics, tetanus antiserum or toxoid, and cryotherapy ${ }^{3}$ are not indicated.

In severe cases when shock persists despite these measures effective antivenom would benefit if it were available. In Great Britain the Standing Medical Advisory Committee has recently agreed that antivenom should no longer be held at designated centres. This is a wise decision as regards the Pasteur antivenom previously recommended, ${ }^{4}$ since this antivenom is unrefined and there is no convincing published evidence of its effectiveness against $V$. berus poisoning. However, recent work ${ }^{5}$ has shown that an antivenom made with the venom of the southern European long-nosed or horned viper, $V$. ammodytes, as sole antigen is both highly refined and remarkably effective in neutralizing $V$. berus venom. This antivenom is obtainable from the Institute of Immunology, Rockefellerova 2, Zagreb, Yugoslavia. If shock is persistent, the contents of five ampoules of this antivenom should be given by intravenous drip, with the precautions recently outlined. ${ }^{6}$ In such cases, if this antivenom is not available, the most suitable treatment would be blood transfusion.

\section{Platelets and Aspirin}

Abnormalities in the functioning of platelets may give rise to bleeding disorders. The platelet dysfunction may be congenital, as in thrombasthenia, or acquired, as in uraemia. ${ }^{12}$ The investigation of a patient with a bleeding tendency starts with a careful history and physical examination and is followed by appropriate laboratory tests. The existence of platelet dysfunction is suggested when the bleeding time is prolonged but the platelet count and general tests of blood coagulation are normal. In such cases it is important to test the bleeding time with special care, and Ivy's method is preferable to Duke's.

A number of tests are now available for investigating platelet function. They include tests of adhesiveness by in vitro and in vivo methods; tests of platelet aggregation using agents such as adenosine diphosphate, collagen, adrenaline, serotonin, and thrombin ; and tests of platelet coagulant function (platelet factor 3 and clot retraction). In thrombasthenia (Glanzmann's syndrome) there is a failure of platelet aggregation by adenosine diphosphate and impaired clot retraction. In uraemia there is a defect in platelet adhesiveness ${ }^{3}$ and in availability of platelet factor 3 . The most recent work on platelet adhesiveness in uraemia has shown that it is inversely correlated with the serum levels of urea and creatinine. ${ }^{4}$

Recently reports have appeared of patients with primary abnormalities of platelets characterized by impairment of aggregation with collagen or adrenaline..$^{5-9}$ It has also been observed that anti-inflammatory drugs, particularly aspirin (acetylsalicylic acid), can induce similar abnormalities of platelet function in healthy persons. ${ }^{10-12} \mathrm{~A}$ single dose of three to four tablets of aspirin (900-1,200 mg.) will induce these abnormalities, which can be detected within a few hours of ingesting the drug and persist for three to seven days. A similar dose of aspirin will also produce a small but distinct increase in the bleeding time in healthy persons and a considerable increase in patients with von Willebrand's disease. ${ }^{13}$ Sodium salicylate does not do so, and it therefore seems likely that the acetyl radicle is important for mediating these changes. Another effect of aspirin on platelets is described by Drs. A. H. Youssef and P. Barkhan in the B.M.F. this week (page 394). They report that aspirin, both in vitro and in vivo, will block the release of heparin-neutralizing activity (platelet factor 4) from human platelets, which normally follows platelet aggregation by adenosine diphosphate or collagen, and they also provide evidence that aspirin attaches to the platelet via the acetyl radicle. This effect of aspirin on platelet function is probably another manifestation of its inhibitory action on the platelet-release reaction in general, involving release also of serotonin and adenosine diphosphate. The exact mechanism of the aspirin effect is unknown, but the changes are perhaps a consequence of its action on the platelet membrane, thus inhibiting release of intrinsic platelet adenosine diphosphate and the platelet granules, which carry serotonin and heparin-neutralizing activity. Interference with platelet function may account for or contribute to the bleeding which may follow the taking of aspirin in some apparently normal persons; and particularly in patients with a pre-existing defect of haemostasis whether congenital, such as haemophilia, von Willebrand's disease, and telangiectasia, or acquired, such as in those on anticoagulant drugs.

A patient recently reported on by $M$. A. Sahud and P. M. Aggeler $^{14}$ is of particular interest in that some of the abnormalities of platelet function were identical with those produced by aspirin ingestion, though the patient had not been taking aspirin and the platelet defect was believed to be of primary type. The similarities and differences between the platelet dysfunction in this patient and that induced by aspirin were investigated in detail. As in the abnormality induced by aspirin, the aggregation of the patient's platelets by trypsin, collagen, and centrifugation was blocked, but aggregation by high concentrations of adenosine diphosphate was normal. Unlike the aspirin defect the patient's bleeding time was very long, in vivo platelet adhesiveness was impaired, adhesion of platelets to collagen fibrils was reduced, and aggregation of platelets by serotonin and glass beads was impaired. Both phases of aggregation induced by adrenaline were blocked, whereas with aspirin only the second phase is abolished. There was also rapid disaggregation of platelets after addition of low concentrations of adenosine diphosphate, N whereas with aspirin the rate of disaggregation was only slightly increased. Platelet transfusions temporarily corrected most of the defects with the exception of adrenaline-induced platelet aggregation.

1 Hardisty, R. M., and Ingram, G. I. C., Bleeding Disorders, 1965. Oxford, Blackwell Scientific Publications.

Stewart, J. H., and Castaldi, P. A., Quarterly fournal of Medicine, $\mathbb{E}$

3 British Medical fournal, 1967, 4, 437.

- Eknoyan, G., Wacksman, S. J., Glueck, H. I., and Will, J. J., Ner England fournal of Medicine, 1969, 280, 677.

s Hirsh, J., Castelan, D. J., and Loder, P. B., Lancet, 1967, 2, 18.

- Hardisty, R. M., and Hutton, R. A., Lancet, 1967, 1, 983.

O'Brien, J. R., Lancet, 1967, 2, 258.

Caen, J. P., Sultan, Y., and Larrieu, M. J., Lancet, 1968, 1, 203.

Cronberg, S., and Nilsson, I. M., Acta Medica Scandinavica, 1968, $183,163$.

10 Weiss, H. J., Aledort, L. M., and Kochwa, S., Fournal of Clinical Investigation, 1968, 47, 2169 .

1 Zucker, M. B., and Peterson, J., Proceedings of the Society for Experimental Biology and Medicine, 1968, 127, 547.

12 O'Brien, J. R., Lancet, 1968, 1, 779

13 Quick, A. J., American Fournal of the Medical Sciences, 1966, 252,

14 Sahud, M. A., and Aggeler, P. M., New England fournal of Medicine, $1969,280,435$. 